\title{
Investigation of correlation between autoimmune antibodies and real-time ultrasound elastosonography scores in patients with Hashimoto's Thyroiditis
}

\section{Li Xiangqi ( $\nabla$ drlixqi@hotmail.com ) \\ Shandong First Medical University CHINA}

Research article

Keywords: Hashimoto's thyroiditis; autoimmune; elastosonography; elastic scores; elastic coefficients

Posted Date: July 12th, 2019

DOI: https://doi.org/10.21203/rs.2.11254/v1

License: (c) (i) This work is licensed under a Creative Commons Attribution 4.0 International License. Read Full License 


\section{Abstract}

Objective To investigate the correlation between autoimmune antibodies, ultrasonic elasticity scores,elasticity coefficients in patients with Hashimoto's thyroiditis.Methods Detected the thyroid function,the thyroid globulin antibody (TgAb) and the titer of thyroid peroxides antibody(TPOAb) by chemiluminescence from the fasting serum of 258 patients suffering with Hashimoto's thyroiditis; The ultrasonic elasticity scores and elasticity coefficients were evaluated after detecting the patients' thyroid with GELOGIQ3 type Color Doppler Ultrasound Diagnostic System and getting a satisfactory elastosonography.Then the correlation between autoimmune antibodies,ultrasonic elasticity scores and elasticity coefficients were analyzed respectively.Results Serum thyroid stimulating hormone (TSH),TGA and TPO in 258 patients with HT increased significantly,the titer of TgAb was positively correlated with elastic coefficients, $r=0.62, P<0.001$, and also positively correlated with elastic scores, $r=0.65, P<0.001 ;$ TPOAb was positively correlated with elastic coefficients, $r=0.63, P<0.001$, and positively correlated with elastic scores as well, $r=0.62, P<0.001$.Conclusions It's helpful to increase the accuracy rate of $\mathrm{HT}$ diagnosis with detection of thyroid autoimmune function combined with Real-time ultrasound elastosonography scores.

\section{Background}

Hashimoto's thyroiditis ( $\mathrm{HT}$ ), also known as chronic lymphocytic thyroiditis,is characterized by lymphocyte infiltration in the thyroid gland and the presence of thyroid-specific autoantibodies in the serum,which is a clinically common autoimmune disease,occupies a high proportion of all thyroid diseases.HT clinical manifestation is complex,in the progression of thyroid inflammatory disease,thyroid function will be hyperthyroidism,normal or decrease with a long duration and poor treatment effect.With the help of thyroid function test and ultrasonic examination,especially the real-time ultrasonic elastic imaging technology,the diagnosis can objectively reflect the hardness of the organization and provide the information of the internal elasticity of the organization,which is known as "electronic palpation".In this study,the ultrasonic elastic images of $258 \mathrm{HT}$ patients were examined,and the correlation between the elastic scores and the elastic strain coefficients,the serum thyroid peroxidase antibody (TPOAb) and the thyroid globulin antibody (TgAb) was investigated.

\section{Methods}

\subsection{General Information}

258 patients with HT in the Second Affiliated Hospital of Shandong First Medical University from January 2016 to December 2016 were confirmed according to Fisher's clinical diagnostic criteria ${ }^{[1]}$ by the serum autoantibodies level,fine needle biopsy and/or pathological examination.Among them,182 female,76 male,age-span $19-74$, average age $(41.29 \pm 11.32)$. $\mathrm{HT}$ is divided into 4 phases ${ }^{[2]}$ according to the results of thyroid function test:The decrease of TSH level and increasing of FT3 or FT4 are shown as hyperthyroidism ; The normal level of TSH,FT3 or FT4 are shown as normal thyroid function;Elevation of 
TSH level,normal FT3 and FT4 level are shown as subclinical hypothyroidism;Elevation of TSH level,decrease of FT3 or FT4 level are shown as clinical hypothyroidism.258 cases of HT patients in this group,52 cases of hyperthyroidism (HT hyperthyroidism group),86 cases of normal thyroid function (HT normal group),64 cases of subclinical hypothyroidism (HT subclinical group), 56 cases of clinical hypothyroidism (HT hypothyroidism group).

Inclusion criteria:Compliance with Fisher diagnostic criteria,the age was between 19 and 73 years old,gender was not limited;Other therapies were discontinued during the study period;The subjects had detailed information about their condition,and the project was examined and approved by the Medical Ethics Committee of the Second Affiliated Hospital of Shandong First Medical University.

Exclusion criteria:Those who do not conform to the above criteria,those who are pregnant or lactating,those who coexist with other diseases during the period of observation, and those who have received other related treatment that may affect the results of this study.

\subsection{Instruments and Methods}

The detector used is color Doppler ultrasonic diagnostic instrument(GE LOGIQ3 type),linear array probe and probe frequency of $7.5-13 \mathrm{MHz}$. The subjects took supine posture,padded the shoulder to make the neck fully exposed,firstly performed routine examination of the two-dimensional ultrasonography,observe thyroid contour,size and thickness of the thyroid gland,the echo of thyroid parenchyma,and the internal blood flow signals of the gland tissue. Then the subjects were told to hold their breath and the twodimensional and elastic images of the thyroid were displayed in real time.The region of interest $(\mathrm{ROI})$ was selected and the probe was manually pressured,the frequency was 2 times/s,and the composite exponent of the pressure vibration frequency next to the elastic image was maintained stable in the range of 34 ,lasted 3 seconds, and relatively stable values and images were obtained.When the pressure is appropriate,the connective tissue around the thyroid capsule is displayed as a continuous banded red,and the muscles around the thyroid gland are uniformly yellowish-green,and the images are relatively clear and stable.On the basis of satisfactory elasticity image,all elasticity images were scored according to the displayed ROI color.Scoring criteria ${ }^{[3]}$ were slightly modified:when the whole or most of the lesion was shown as green,marked as 1 point; when the center of the lesion was green and blue,marked as 2 points based upon green;when the lesion range was shown as green and blue with a similar proportion,marked 3 points when the overall focus of the lesion was blue or a few green inside,marked 4 points; when the lesions and surrounding tissues were shown as blue with or without green in the internal,marked 5 points.

The dual real-time display function of color Doppler ultrasound was enabled again,the images were replayed,the elastic images were observed and analyzed,a frame of relatively stable images was selected,the regions of interest were drawn on the two-dimensional images,and $\mathrm{HT}$ thyroid parenchyma lesions area were set as (A).The ipsilateral sternocleidomastoid muscle (B) was used as the reference area.The average elastic strain data of the ROI region are calculated by the instrument system. Taking the ratio of the two,that is the elastic strain rate of the lesion region (SR),or the elastic coefficients. The image 
scores and SR were measured by double blind method by two ultrasound diagnostics,and comprehensive evaluation was performed by the third ultrasonic doctor with diagnostic qualification when there are different opinions.

Thyroid function test:Early morning fasting, $3 \mathrm{~mL}$ venous blood was collected in vacuum nonanticoagulant test tube,serum was separated with $1500 \mathrm{r} / \mathrm{min}$ speed,centrifuging for $10 \mathrm{~min}$. The serum sample test must be accomplished on the same day. The thyroid function,the thyroid peroxidase antibody (TPOAb) and the thyroglobulin antibody ( $\mathrm{TgAb}$ ) titer were detected by the chemiluminescence method. The reference range drawn up by the laboratory of the Second Affiliated Hospital of Shandong First Medical University is as follows: FT3 $1.45-3.48 \mathrm{pg} / \mathrm{mL}, \mathrm{FT} 40.71-1.85 \mathrm{ng} / \mathrm{mL}$, TSH 0.35-

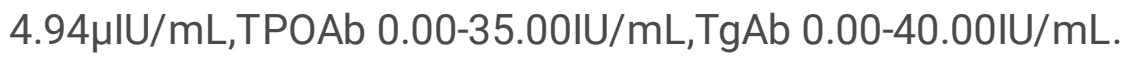

\subsection{Statistical Analysis}

SPSS 17 statistical analysis software was used to analyze the data.Measurement data was represented by $\pm s$. The comparison of data between groups is based on the nature of data.T-Test or variance analysis was used.The correlation between elastic image scores,elastic strain coefficients and thyroid function was analyzed by Spearman correlation analysis.P value 0.05 was considered statistically significant .

\section{Results}

\subsection{Thyroid function and autoimmune antibodies in different HT groups}

The serum levels of TgAb,TPOAb and TSH were increased in 258 patients with HT.TSH was in the normal range in Hyperthyroidism group and Normal group,but there were significant differences among the groups $(\mathrm{P}<0.01)$.It showed that with the decrease of FT3 and FT4,TgAb,TPOAb and TSH were increasing gradually, $\mathrm{P}<0.05$ or 0.01 . The results were shown in Table 1.

\subsection{Ultrasonic Elastic Imaging scores and Elastic coefficients in different HT groups}

There were significant differences in SR among the other groups except the SR of the HT hyperthyroidism group and HT normal group, $\mathrm{P}<0.01$ or $\mathrm{P}<0.05$ (Subclinical group vs Hypothyroidism group).In other words, with the hypothyroidism, the SR of thyroid tissue increased. The elastic scores of thyroid was significant differences between the other groups except the HT hyperthyroidism group and the HT normal group, $\mathrm{P}<0.01$ \Hyperthyroidism group vs Hypothyroidism group; Normal group vs Hypothyroidism group $₫$ or $\mathrm{P}<0.05 \bigotimes$ Hyperthyroidism group vs Subclinical group; Normal group vs

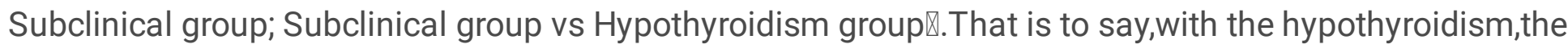
elastic scores of thyroid tissue increased, which indicated that the hardness of thyroid increased.The results were shown in Table 2 and Figure 1-4.

\subsection{Correlation between thyroid Ultrasound elastic scores,elastic Coefficients and autoantibodies in HT patients}


The titer of TgAb was positively correlated with SR in 258 patients $(r=0.62, P<0.001)$; There was a positive correlation between $\operatorname{TgAb}$ and elasticity scores $(r=0.65, P<0.001)$;TPOAb and $S R$ was positively correlated $(r=0.63, P<0.001)$ as well as TPOAb and elastic scores $(r=0.62, P<0.001)$. The results were shown in Figure 5-8.

\section{Discussion}

$\mathrm{HT}$ is a kind of common clinical autoimmune thyroid disease,the etiology is no clear,the clinical manifestations are various,iodine intake is an important environmental factor affecting the development of the disease.In recent years, with the popularization of iodized salt,iodine intake gradually increased,the incidence of this disease increased significantly ${ }^{[4]}$.The typical pathological changes are the infiltration of a large number of lymphocytes and the proliferation of connective tissue in different degrees. High autoantibodies titer of thyroid-resistant components can be detected in the patients' serum,such as TPOAb and TgAb.Because the disease is more obscure,the patients don't come to see a doctor until hyperthyroidism or hypothyroidism symptoms exists or histological diagnosis was confirmed due to the operation of thyroid nodule of tumor ${ }^{[5]}$.

HT may have no clinical symptoms in the early stage,only TPOAb and TgAb elevated,hyperthyroidism may also appears in the early stage,then feel weak,hypothyroidism appears in the late stage.HT-related

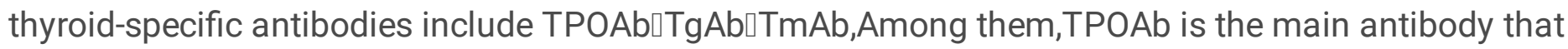
mediates the cytotoxic effect of cell-mediated antibody.On the basis of T-cell mediated cell damage,thyroid follicular epithelial cells are further damaged,resulting in hypothyroidism.TPO is an indispensable enzyme in the synthesis of thyroid hormones, and normally TPO does not overflow into the blood from the thyroid gland. When the structure of thyroid follicular cells were destroyed,TPO leaks from the top of the thyroid cell,namely the edge of the cavity of the thyroid follicular cell out of the peripheral blood.As an important autoantigen for autoimmune thyroid diseases,TPO stimulated the body's immune system,producing thyroid tissue component antibody TPOAb and activating complement and antibody dependent cell mediated cytotoxicity,resulting in thyroid immune damage ${ }^{[6]}$. With the discovery of new effector T-cell subsets and the further understanding of T-cell subsets, it was believed that thyroid organspecific autoimmune diseases were mainly related to Th1 cells ${ }^{[7]}$.Th17 cells,as independent CD4 T-cell subsets,played a pathogenic role by releasing IL-17,IL-17F and IL-22.It could induce local cells to synthesize chemical factors and pro-inflammatory factors, and affect the production of autoimmune antibodies. The abnormal secretion of IL-17 may lead to the disorder of thyroid autoimmune function and then induce the occurrence and development of $\mathrm{HT}^{[8,9]}$. It had also been confirmed that the immune imbalance of peripheral blood Treg/Th17 cell axis ran through the different stages of HT,and had been abnormal in the normal thyroid function stage.A qualitative change began to appear in subclinical hypothyroidism and the ratio of Th17/Treg cells were positively correlated with the thyroid autoantibody TPOAb-TgAb.The results suggested that the imbalance of Th17/Treg cell axis might play an important role in the production of thyroid autoantibodies and the abnormal immune response of T-cells mediated by thyroid autoantibodies, thus participated in the autoimmune injury of thyroid tissues ${ }^{[10]}$.However,up- 
regulation of IL-35/IL-35 expression mediated by inducible T-cells to induce Treg immunosuppression might play an important role in the autoimmune model ${ }^{[11]}$.

The normal thyroid tissue is composed of follicular structure and interfollicular connective tissues. The follicular cavity contains colloid and soft texture. However,due to the infiltration of lymphocytes and plasma cells in HT glands,the normal follicles were destroyed or even follicular structures were atrophied and tissue fibrosis,as a result,the thyroid hardness was gradually increased.At present,the first choice of imaging methods for thyroid disease is ultrasonography,which is widely used in clinic.Real-time ultrasonic elastic imaging is a new ultrasonic diagnosis technique,which can provide objectively some information on the internal hardness characteristic,and it was first proposed by Ophir et al[ ${ }^{[12]}$ in 1991. The basic principle is based on the different elastic coefficients (stress/strain) of various tissues,the tissues structure will deform in different degrees after being compressed by local external force,then transform the amplitude of ECHO signal into real-time color images before and after compression,with the help of color change to reflect the hardness of organization. Real-time ultrasound-elastic imaging of the thyroid,which indirectly responds to the hardness of the thyroid parenchyma by measuring SR,provides a new auxiliary information for HT ultrasound diagnosis,and it can better assess $\mathrm{HT}$ function status and pathological progression as a supplement of conventional ultrasonography.

TGAb and TPOAb are the signature antibodies of Hashimoto's thyroiditis, which is convenient,accurate and sensitive,which is of great significance for diagnosis,prognosis and follow-up after treatment.Ultrasound examination combined with serological determination of thyroid autoimmune antibodies and thyroxine level,although it can be clearly diagnosed with $\mathrm{HT}$,However,in the conventional two-dimensional ultrasound examination,there is a lack of objective quantitative indicators in response to the progress of the disease.Real-time ultrasound elastography combined with thyroid autoimmune function can improve the diagnosis of Hashimoto's thyroiditis.

The results of the study showed that the SR and elasticity score of thyroid tissue increased due to the increase of TGAb and TPOAb in serum of HT patients.It indicated that the persistence or further increase of TGA and TPO led to the increase of hardness of thyroid tissues. Therefore,in clinical work,once the thyroid tissue is hard,TgAb-TPOAb and thyroid ultrasound examination should be performed to make a definite diagnosis and provide the evidence for clinical treatment.

\section{Declarations}

\section{Acknowledgements}

This work was supported by the National Natural Science Foundation of China (No.81473687), and Natural Science Foundation of Shandong Province, China (No.ZR2009CM039 and No. ZR2013HM038), and Health Science and Technology Subject of Shandong Province (No. 2016BJ0015), and Tai'an Science and Technology plan (No.201540186).

\section{Funding}


The authors thank Tai'an Science and Technology plan (No.201540186) for funding the project.

\section{Compliance with ethical standards}

Conflict of interset All authors report no potential conflict of interest

Ethical approval The study was approved by Second Affiliated Hospital of Shandong First Medical University. All procedures performed in study involving human participants were in accordance with the ethical standards of the institutional and national research committee and with the 1964 Helsinki declaration and its later amendments.

Informed consent Informed consent was obtained from all individual participants included in the study for patients' study participation and publication of identifying information and images.

Open Access This article is distributed under the terms of the Creative Commons Attribution 4.0 International License (http://creativecommons.org/licenses/by/4.0/), which permits unrestricted use, distribution, and reproduction in any medium,provided you give appropriate credit to the original author(s) and the source, provide a link to the Creative Commons license, and indicate if changes were made.

Address Correspondence to: Dr. Xiangqi Li, Department of Breast surgery, Second Affiliated Hospital of Shandong First Medical University, 706 Taishan Road, Tai'an 271000, China. Tel: +86-538-6237727; Fax: +86-538-8420042; E-mail: drlixqi@hotmail.com;

Ying Wang, Department of Rehabilitation medicine, Second Affiliated Hospital of Shandong First Medical University, 706 Taishan Road, Tai'an 271000, China. E-mail: drlixqi@163.com

\section{References}

[1] Fisher DA,Oddie TH,Johnson DE,et al.The diagnosis of Hashimoto's thyroiditis [J].Journal of Clinical Endocrinology and Metabolism,1975,40:795-801.

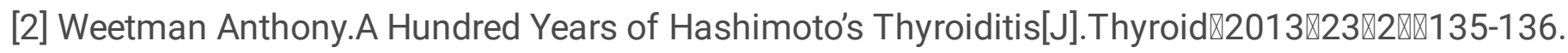

[3] Itoh Ako, Ueno Ei,Tohno Eriko,et al.Breast disease:clinical application of US elastography for diagnosis[J].Radiology,2006,239 (2):341-350.

[4] Langer P,Tajtakova M,Kocan A,et al.Thyroid volume,iodine intake,autoimmune thyroid disorders,inborn factors,and endocrine disruptors:twenty -year studies of multiple effects puzzle in Slovakia[J]区Endocr $\otimes$ egul,2012,46(4):191-203】

[5] Bektas Uysal Hilal,Ayhan Mediha.Autoimmune affects health-related quality of life in patients with Hashimoto's thyroiditis[J].The Kaohsiung journal of medical sciences,2016,32(8):427-433. 
[6] Zhao C,Gao Y,Zhao L,et al.The expression and function of the neonatal Fc receptor in thyrocytes of Hashimoto's thyroiditis[J].Int Immunopharmacol,2017,3(44):53-60.

[7] Zhu J,Yamane H,Paul WE.Differentiation of effector CD4 T cell populations[J].Annu Rev Immunol,2010,28:445-489.

[8] Lovett-囚acke AE,Yang Y,囚acke MK囚 Th1 versus Th17:are T cell cytokines relevant in multiple sclerosis? [J].Biochim Biophys Acta,2011,1812(2):246-251区

[9] Fatemeh Esfahanian; Roghayeh Ghelich; Hoda Rashidian,et al.Increased levels of serum interleukin-17 in patients with Hashimoto's thyroiditis[J].Indian Journal of Endocrinology and Metabolism,2017,21(4):551-554.

[10] Lee YK,Mukasa R,Hatton RD,et al.Developmental plasticity of Th17 and Treg cells[J].Curr Opin Immunol,2009,21(3):274-280.

[11] Yilmaz H, Cakmak M, Ceydilek B, et al.Role of interlekin-35 as a biomarker in patients with newly diagnosed Hashimoto's thyroiditis [J].Endocr Regul.,2016,50(2):56-61.

[12] Ophir J,Cespedes I,Ponnekanti H,et al.Elastography:a quantitative method for imaging the elasticity of biological tissues[J].Ultrasonic Imaging,1991,13囚2囚:111-134.

\section{Tables}

Table 1 Comparison of thyroid function and autoimmune antibodies indifferent HT groups

\begin{tabular}{lclllll}
\hline Groups & $\mathrm{n}$ & $\begin{array}{l}\mathrm{FT3} \\
(\mathrm{pg} / \mathrm{mL})\end{array}$ & $\mathrm{FT4}(\mathrm{ng} / \mathrm{mL})$ & $\mathrm{TSH}(\mathrm{\mu IU} / \mathrm{mL})$ & $\mathrm{TgAb}(\mathrm{IU} / \mathrm{mL})$ & $\mathrm{TPOAb}(\mathrm{IU} / \mathrm{mL})$ \\
\hline $\begin{array}{l}\text { Hyperthyroidism } \\
\text { group }\end{array}$ & 52 & $4.32 \pm 0.36$ & $2.19 \pm 0.27$ & $0.19 \pm 0.13$ & $98.62 \pm 21.36$ & $119.59 \pm 98.36$ \\
Normal group & 86 & $2.74 \pm 0.26$ & $1.58 \pm 0.34$ & $2.75 \pm 0.62$ & $128.36 \pm 31.12$ & $187.18 \pm 79.46$ \\
Subclinical group & 64 & $2.36 \pm 0.31$ & $1.04 \pm 0.25$ & $5.68 \pm 0.56$ & $169.67 \pm 36.54$ & $256.68 \pm 102.38$ \\
$\begin{array}{l}\text { Hypothyroidism } \\
\text { group }\end{array}$ & 56 & $1.26 \pm 0.32$ & $0.64 \pm 0.27$ & $10.78 \pm 1.02$ & $261.12 \pm 42.26$ & $329.48 \pm 158.12$ \\
HT group & 258 & $3.16 \pm 2.14$ & $0.98 \pm 0.56$ & $8.52 \pm 3.14$ & $181.66 \pm 124.39$ & $254.88 \pm 186.05$ \\
\hline
\end{tabular}

Table 2 Comparison of ultrasonic elasticity imaging scores and elastic coefficients in different HT groups 


\begin{tabular}{llcc}
\hline Groups & $\mathrm{n}$ & Strain ratio $₫ \mathrm{SR}$ & Elasticity score \\
\hline Hyperthyroidism group & 52 & $1.53 \pm 1.12$ & $1.73 \pm 1.36$ \\
Normal group & 86 & $1.86 \pm 1.26$ & $1.92 \pm 1.38$ \\
Subclinical group & 64 & $2.28 \pm 1.42$ & $2.39 \pm 1.27$ \\
Hypothyroidism group & 56 & $2.69 \pm 1.25$ & $2.92 \pm 1.29$ \\
HT group & 258 & $2.32 \pm 1.35$ & $2.51 \pm 1.37$ \\
\hline
\end{tabular}

\section{Figures}

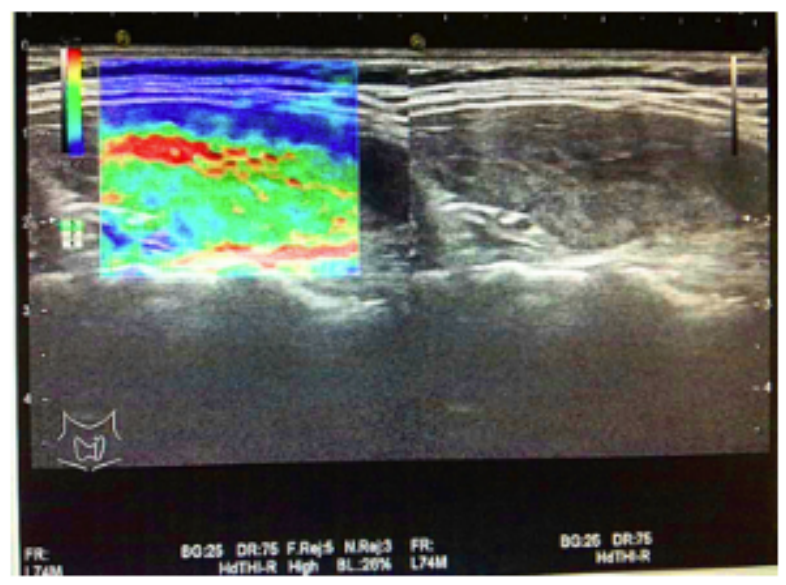

\section{Figure 1}

Hyperthyroidism groups Elastic score 1 point

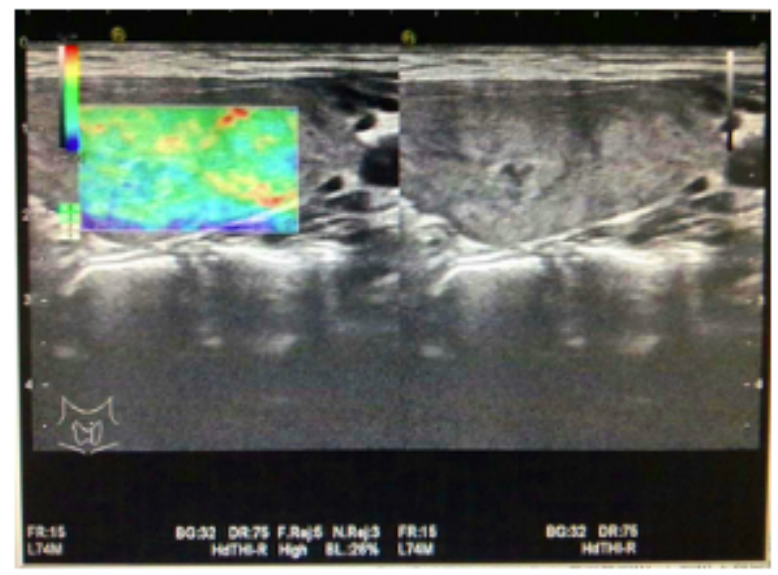

Figure 2

Normal groups Elastic score 2 points 


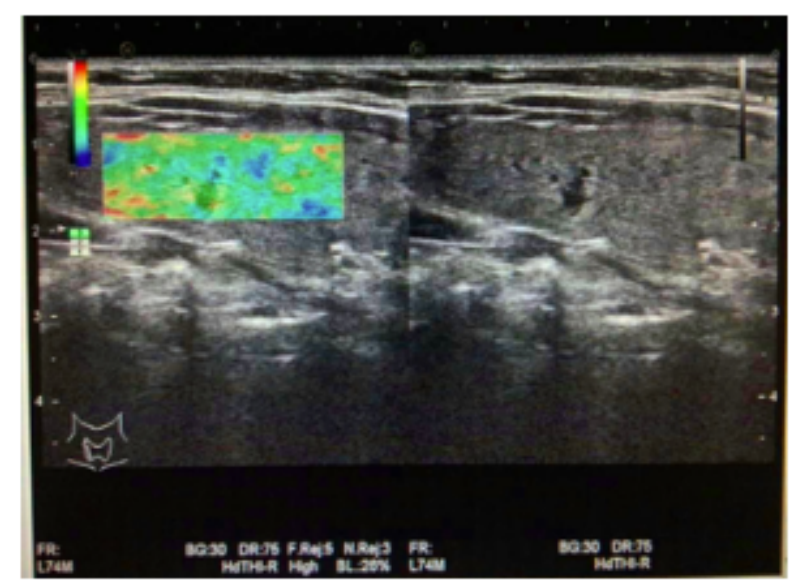

Figure 3

Subclinical groups, Elastic score 3 points

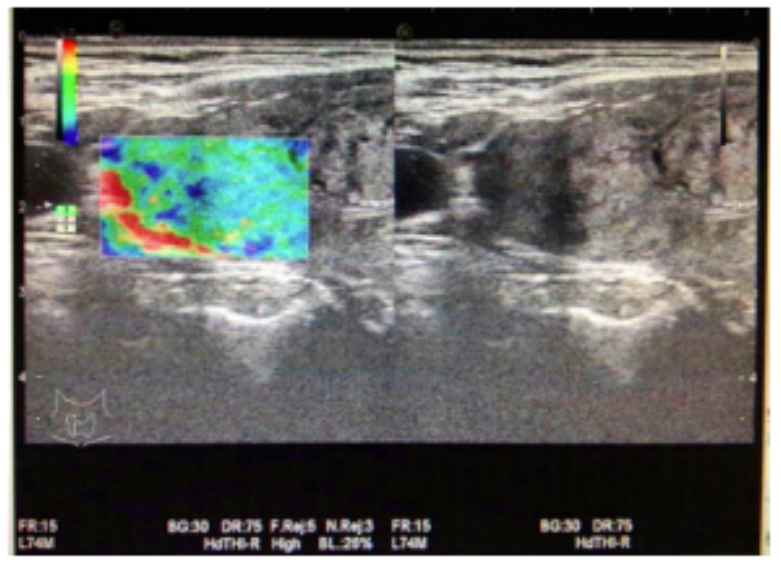

Figure 4

Hypothyroidism groups Elastic score 4 points

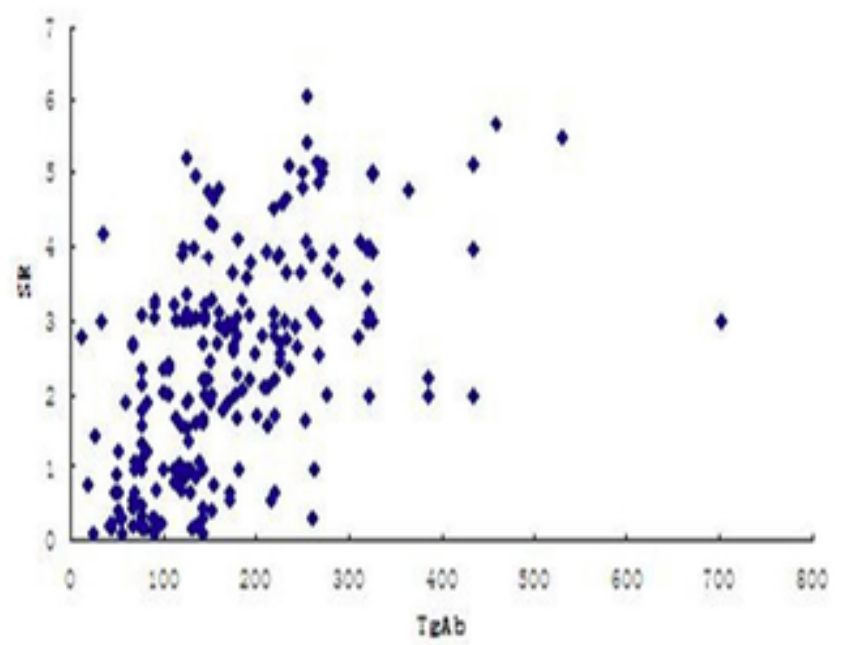


Figure 5

Correlation between $\mathrm{TgAb}$ and SR

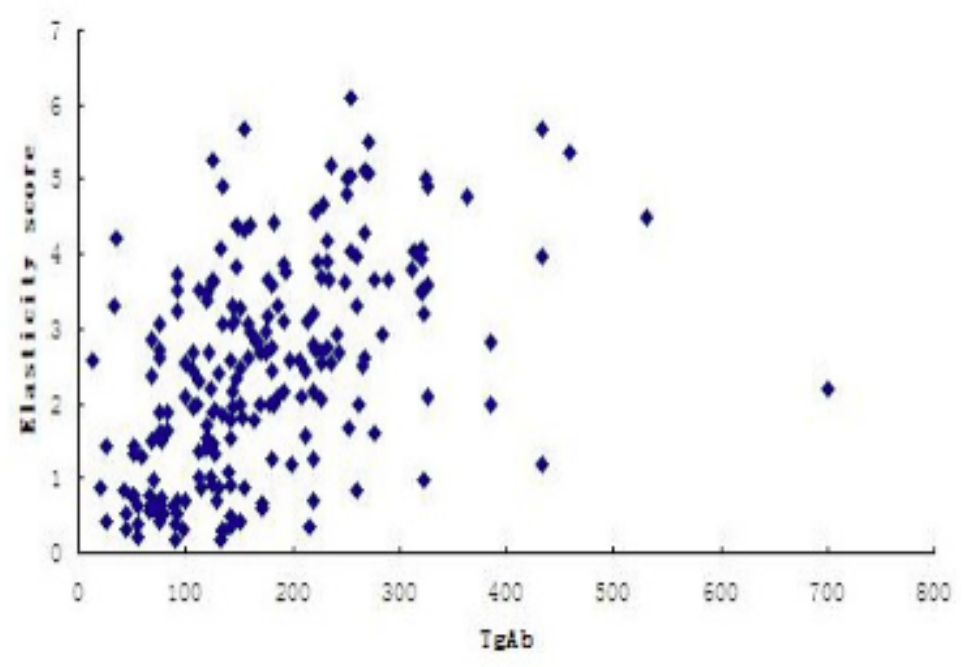

Figure 6

Correlation between $\operatorname{TgAb}$ and elastic coefficients

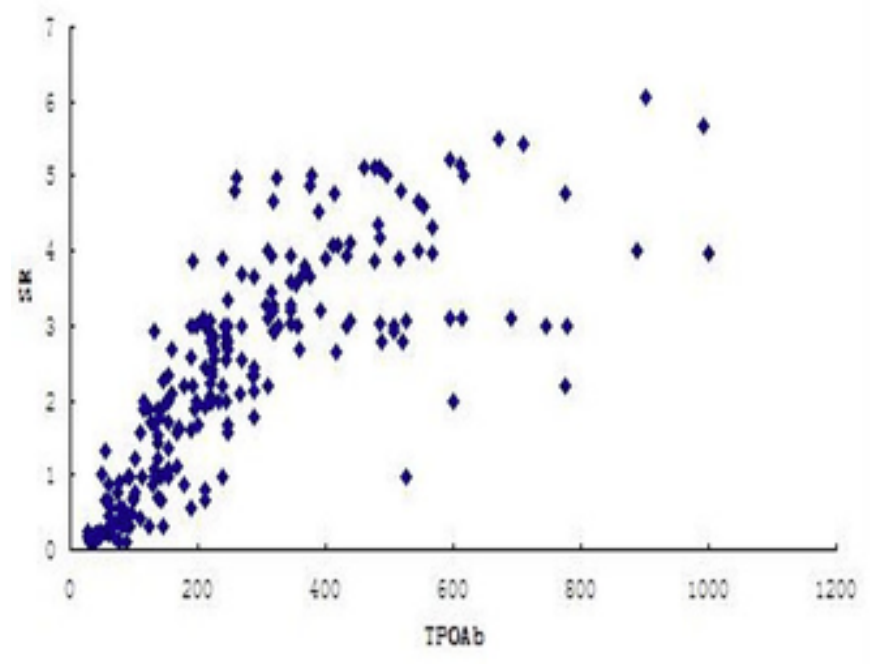

Figure 7

Correlation between TPOAb and SR 


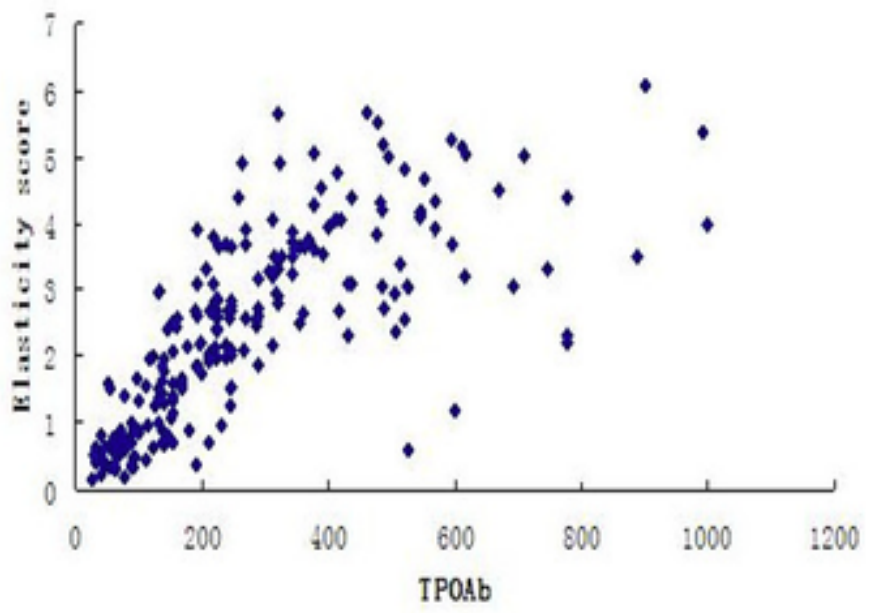

Figure 8

Correlation between TPOAb and elastic scores 